\title{
COVID-19 Vaccination Rates Are Lowest among Political Outsiders in the United States
}

$$
\text { Byungkyu Lee } \mathrm{a}^{*+} \text { and James Chu }{ }^{\mathrm{b+}}
$$

${ }^{\mathrm{a} D e p a r t m e n t}$ of Sociology, Indiana University; ${ }^{\mathrm{b}}$ Department of Sociology, Columbia University

* To whom correspondence should be addressed. E-mail: bl11@indiana.edu

+ Both authors equally contributed to this article.

\begin{abstract}
Vaccine hesitancy is a critical barrier to widespread vaccination uptake and containment of the COVID-19 pandemic. In the United States, vaccines have become politically polarized, with high rates of vaccine hesitancy observed among Republicans. In contrast to prior research focusing on partisan gaps, we investigate vaccination attitudes and uptake among a group overlooked in prior research: those who are eligible to vote but did not register in the presidential elections. Drawing on nationally representative and longitudinal survey data from April 2020 to October 2021, we show that this group - whom we call "political outsiders" - represents about $16 \%$ of the U.S. population. They had the lowest vaccination rate (47\%) by 2021 October, significantly lower than Republican (65\%), Independent (76\%), and Democratic voters (88\%). Further, we find that political outsiders are less likely to trust physicians compared to other partisan groups. Because the sources they trust differ from partisans, existing public health messaging may be less likely to reach them successfully. Finally, we find that political outsiders experience more socio-economic hardships and are less integrated into society. Hence, our results underscore the importance of targeted efforts to reach this highly vulnerable population.
\end{abstract}

Keywords: COVID-19; vaccination; polarization; political outsiders 


\section{Introduction}

Mass vaccination is the most effective way to contain the COVID-19 pandemic, but vaccine hesitancy remains a serious challenge in many countries (1). In the United States, vaccine uptake has grown increasingly polarized along political lines, with high rates of vaccine hesitancy observed among Republicans $(2,3)$. Scholars and policymakers have sought to identify strategies to promote vaccine uptake among vaccine hesitant groups $(4,5)$, but recent work has overlooked a large group of the U.S. population: those who do not register to vote.

We call non-voters who withdraw from participation in political institutions "political outsiders." In contrast to Independents who avoid identifying as partisan but still participate in elections (6), political outsiders reject identification with existing political categories and avoid voting, in part because of their distrust in existing institutions (7). They are also less likely to be socially integrated in groups that would encourage political engagement (8). We anticipate that political outsiders may show stronger vaccine hesitancy than partisans, given that trust in medical institutions is a key determinant for vaccination and integration in social groups motivates people to get vaccinated to protect others $(9,10)$. Thus, failure to distinguish this group may conceal a sizable population that are hesitant to get vaccinated.

We investigate overall levels of COVID-19 vaccine uptake and patterns of trust among political outsiders using data from the Understanding America Survey (UAS), a nationally representative and longitudinal survey, from 2020 April to 2021 October (11). We identify political outsiders by those who do not register to vote, although the results are similar if we use an alternative definition of political outsiders (i.e., those who refuse to identify themselves using liberal-conservative ideological labels). Our analysis includes 7,814 respondents; among them, 
about $36 \%$ registered to vote as Democrats, $28 \%$ registered as Republicans, $20 \%$ registered as Independent and other parties, and $16 \%$ did not register to vote.

\section{Results}

Figure 1, Panel A shows high levels of political polarization in COVID-19 vaccination, with $87.5 \%$ of Democratic voters receiving at least one dose of a COVID-19 vaccine by October 2021, compared to only $65.2 \%$ of Republican voters. Trends for Independents fall between Republicans and Democrats $(76.3 \%$ by October 2021$)$, and political outsiders report the lowest vaccination rates, with only $47.4 \%$ of them receiving vaccines by October 2021. Similarly, political outsiders have demonstrated the highest levels of COVID-19 vaccine hesitancy throughout the COVID-19 pandemic (in Panel B). Unlike the polarization of vaccine attitudes between Democrats and Republicans after the 2020 November presidential election, political outsiders showed high levels of vaccine hesitancy initially and remained hesitant throughout the pandemic period.

Figure 1, Panel C shows that the prevalence of COVID-19 infections was comparable between political outsiders and Republicans, meaning that the differences in COVID-19 prevalence cannot explain low vaccination rates among political outsiders. In fact, low vaccination rates among Republicans and political outsiders may be responsible for the surge in COVID-19 infections among these groups in the fall of 2021, when many social distancing policies were rolled back. Figure 1, Panel D shows that their low vaccination rates cannot be explained by low perceptions of COVID-19 risk, given that political outsiders consistently have perceived higher levels of COVID-risk (i.e., chance of being hospitalized if a person gets COVID-19) than other groups. 
Low vaccination rates among political outsiders, despite high levels of perceived COVID-19 risk, may be explained in part by the extent to which they trust different sources of information about the coronavirus. Figure 2 shows that Democrats are more likely to trust physicians, CDC, Biden, and MSNBC; Republicans are more likely to trust Trump and Fox News than other groups; and Independents and political outsiders display middling levels of trust in polarized sources like Trump, Biden, Fox, and MSNBC. The exception to this general pattern is that political outsiders are the least likely to trust physicians than any other groups. Across all sources of COVID-19 information, the mean levels of trust expressed by political outsiders was 0.93 on a 0 to 3-point scale, compared to 0.95 for Republicans, 0.99 for Independents, and 1.18 for Democrats. This low level of trust, both overall and especially in physicians, may partially explain why political outsiders are hesitant to get vaccinated, given that medical experts are typically the most authoritative source for accurate vaccination information.

Aside from a lack of institutional trust, political outsiders may face additional barriers to vaccine access, such as inconvenient vaccine appointments, as a result of their socio-economic vulnerability (12). Compared to those who register to vote, political outsiders are more likely than partisans to be female $(8.8 \%$; $95 \%$ CI: $-10.4 \%$ to $-7.2 \%)$, younger ( -9.2 years; $95 \%$ CI: -9.7 to -8.7 years), non-White $(-7.7 \%, 95 \%$ CI: $-9.3 \%$ to $-6.1 \%)$, less educated (-1.7 education years, 95\% CI: -1.8 years to -1.6 years), more likely to be evicted (2.2\%, 95\% CI: $1.8 \%$ to $2.7 \%)$, less likely to be employed (-9.1\%, $95 \%$ CI: $-10.7 \%$ to $-7.5 \%)$, and less likely to have health insurance $(-21.6 \%, 95 \%$ CI: $-23.2 \%$ to $-20.1 \%)$. Finally, political outsiders may have a weaker desire to protect others in the community because they are socially isolated (9). In accordance with this possibility, political outsiders are less likely to be married (-20.7\%, 95\% CI: $-22.2 \%$ to $-19.2 \%)$, and have smaller acquaintance networks (-6.1 persons, $95 \%$ CI: -7.2 to -5.1 persons). 


\section{Discussion}

Political partisanship has emerged as one of the strongest predictors of Americans' response to the COVID-19 pandemic $(13,14)$. Our paper replicates prior research on the partisan gaps between Republicans and Democrats, but our results indicate that political outsiders, who did not register to vote in the presidential election, have the lowest COVID-19 vaccination rates, even as they had the highest COVID-19 infection rates by 2021 October.

Our results imply, first, that concern over COVID-19 risk and willingness to get vaccinated need not be correlated. Polarization has led many Republican voters to downplay the threat of COVID-19, but political outsiders remain highly concerned about the pandemic risk than Republicans. Despite high levels of concern, they are more skeptical of vaccines than Republicans. While future work is needed to identify exactly why this is the case, our results suggest that their uniquely low levels of trust in physicians, their socio-economic vulnerability, and their lack of social integration are likely contributors to high rates of vaccine hesitancy.

Second, our findings about trusted sources suggest that current public health efforts may not be effective among political outsiders, who trust in different sources when compared to other partisan groups. For instance, the use of elite cues, such as vaccine endorsements from Trump and elite Republican politicians, will be unlikely to sway those who chose not to vote due to their distrust in politicians. Moreover, the lower level of trust in physicians among political outsiders (compared to other groups) suggests that promoting vaccination through local health professionals will be less effective for them.

Finally, we show that COVID-19 pandemic attitudes and behaviors of political outsiders, who do not vote, are systematically different from those of Independents, who remain engaged in the political process through voting. Although research has shown that independents avoid 
identifying themselves with existing partisan categories due to their disdain for political parties (6), their vaccination rates are not as low as political outsiders, who entirely withdraw from the political process. At a broader level, research seeking to address problems of vaccine hesitancy has largely focused on partisan divides in COVID-19 vaccination rates in the US $(3,5)$ but overlooked political outsiders. While researchers often categorize non-voters, independent voters, and third-party voters together as "Independents" or "Other", our results imply that this practice obscures political outsiders -- a distinctive and vulnerable group with uniquely low rates of vaccine uptake.

\section{Materials and Methods}

We use data from April 2020 to October 2021 from the Understanding America Survey (UAS), a probability-based Internet panel of American households. The UAS respondents recruited through Address Based Sampling are individuals aged 18 and older who answer surveys via an online interface; internet-connected tablets are provided to households if necessary (11). We measure COVID-19 vaccine uptake, COVID-19 vaccine hesitancy, COVID-19 positivity, COVID-19 risk assessment. In addition, respondents are asked how much they trust different sources of information for COVID-19. We classify respondents' party registration status into Democrats, Republicans, Independents, and political outsiders (i.e., those who did not vote, see SI Appendix for more details). We use survey weights provided by the UAS in all analyses.

Data Availability: All analysis codes to replicate results will be made available at https://dataverse.harvard.edu/dataverse/bk. All data used in this study are available for download for any registered UAS data user from https://uasdata.usc.edu/index.php. 


\section{Acknowledgment}

We thank Peter Bearman, Jamie Druckman, Elaine Hernandez, Barum Park and Kosali Simon for their helpful comments. Financial support from the American Assembly at Columbia University is gratefully acknowledged. The project described in this paper relies on data from survey(s) administered by the Understanding America Study, which is maintained by the Center for Economic and Social Research (CESR) at the University of Southern California. The content of this paper is solely the responsibility of the authors and does not necessarily represent the official views of USC or UAS.

\section{References}

1. J. S. Lavine, O. N. Bjornstad, R. Antia, Immunological characteristics govern the transition of COVID-19 to endemicity. Science 371, 741-745 (2021).

2. P. G. Szilagyi, et al., Likelihood of COVID-19 vaccination by subgroups across the US: post-election trends and disparities. Hum. Vaccines Immunother. 17, 3262-3267 (2021).

3. K. Viswanath, et al., Individual and social determinants of COVID-19 vaccine uptake. BMC Public Health 21, 818 (2021).

4. S. E. Bokemper, G. A. Huber, A. S. Gerber, E. K. James, S. B. Omer, Timing of COVID-19 vaccine approval and endorsement by public figures. Vaccine 39, 825-829 (2021).

5. S. L. Pink, J. Chu, J. N. Druckman, D. G. Rand, R. Willer, Elite party cues increase vaccination intentions among Republicans. Proc. Natl. Acad. Sci. 118 (2021).

6. S. Klar, Y. Krupnikov, Independent Politics: How American Disdain for Parties Leads to Political Inaction (Cambridge University Press, 2016) https:/doi.org/10.1017/CBO9781316471050 (November 27, 2021).

7. R. Herrera, Understanding the Language of Politics: A Study of Elites and Masses. Polit. Sci. Q. 111, 619-637 (1996).

8. H. E. Brady, S. Verba, K. L. Schlozman, Beyond SES: A Resource Model of Political Participation. Am. Polit. Sci. Rev. 89, 271-294 (1995).

9. L. Korn, R. Böhm, N. W. Meier, C. Betsch, Vaccination as a social contract. Proc. Natl. Acad. Sci. 117, 14890-14899 (2020). 
10. O. Yaqub, S. Castle-Clarke, N. Sevdalis, J. Chataway, Attitudes to vaccination: A critical review. Soc. Sci. Med. 112, 1-11 (2014).

11. A. Kapteyn, et al., Tracking the Effect of the COVID-19 Pandemic on the Lives of American Households. Surv. Res. Methods 14, 179-186 (2020).

12. K. W. Strully, T.-C. Yang, County Social Vulnerability and Influenza Vaccine Rates: National and Local Estimates for Medicare Recipients. Am. J. Prev. Med. (2021) https:/doi.org/10.1016/j.amepre.2021.06.015 (December 6, 2021).

13. J. Clinton, J. Cohen, J. Lapinski, M. Trussler, Partisan pandemic: How partisanship and public health concerns affect individuals' social mobility during COVID-19. Sci. Adv., eabd7204 (2020).

14. J. N. Druckman, S. Klar, Y. Krupnikov, M. Levendusky, J. B. Ryan, Affective polarization, local contexts and public opinion in America. Nat. Hum. Behav. 5, 28-38 (2021). 


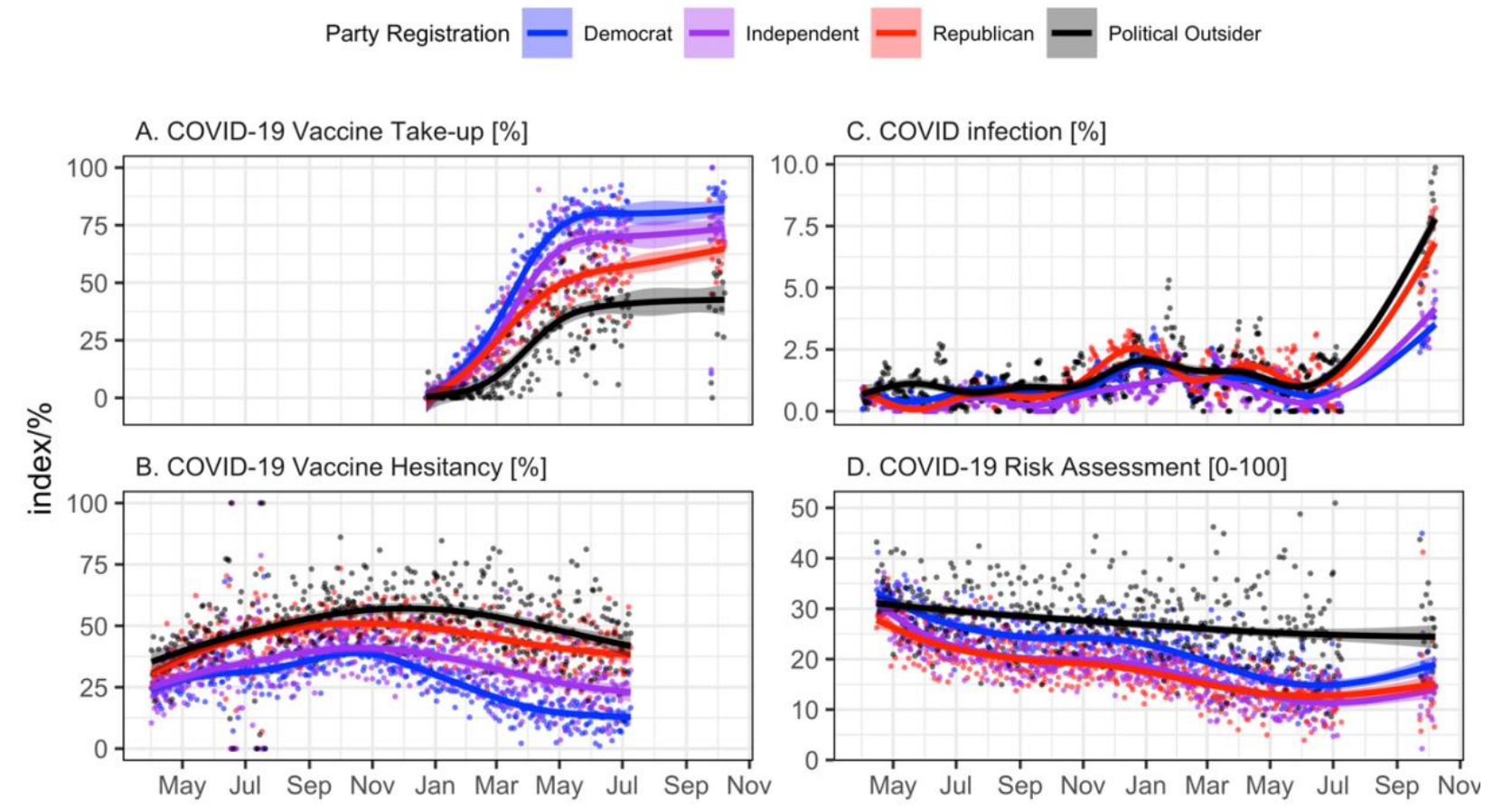

Figure 1. Trends for COVID-19 Vaccine and Other Outcomes by Party Registration

Status. Each dot represents daily mean estimates adjusted by the final survey weights except for COVID positive rates that were estimated by 7 day moving average. We use generalized additive models (GAM) to estimate trends for different subgroups. 


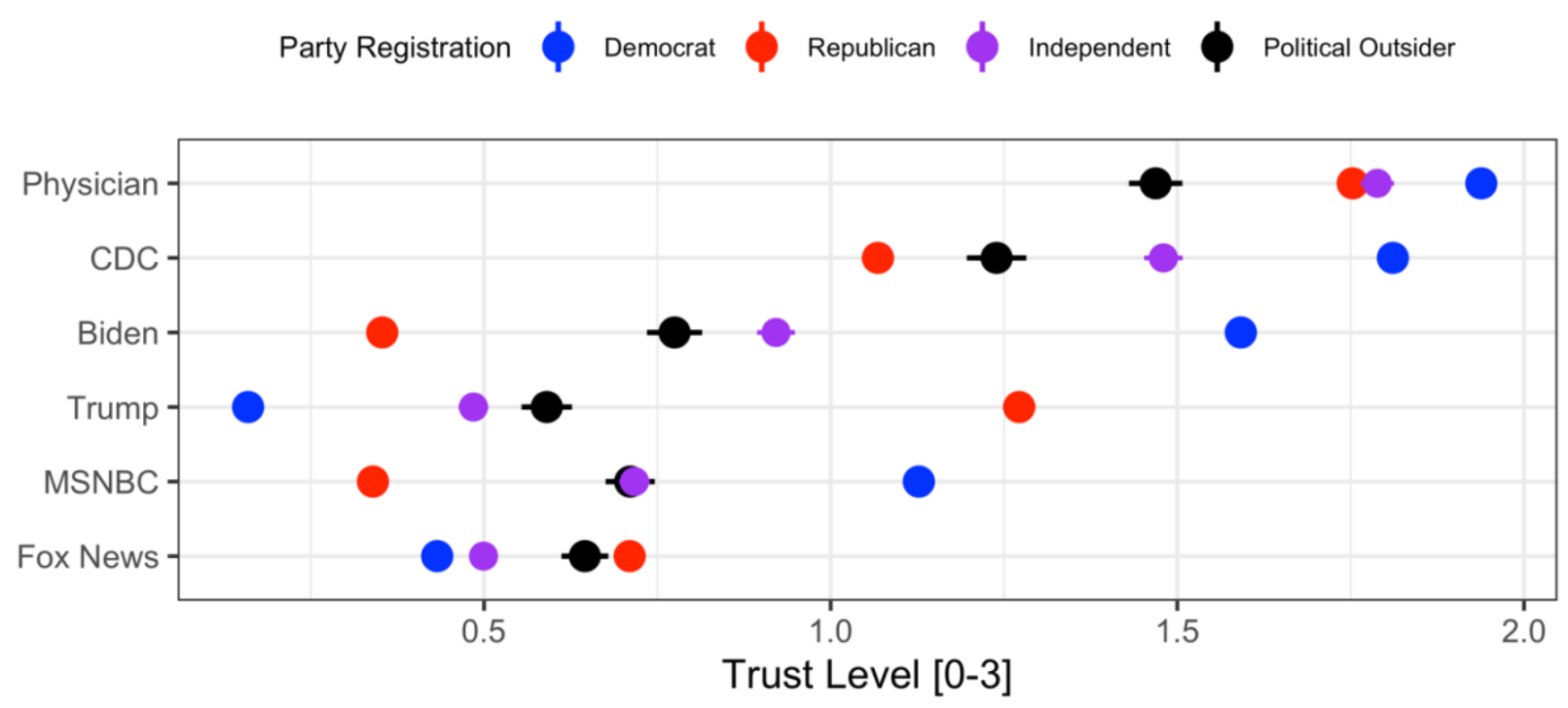

How much do you trust the following sources of information about the coronavirus?

Figure 2. Trust in the Sources of Information about COVID-19 by Party Registration

Status. Each dot represents weighted mean estimates with 95\% confidence intervals across the whole period for different subgroups. Survey responses are coded into Do not trust at all $(=0) /$ Trust somewhat $(=1) /$ Trust mostly $(=2) /$ Trust completely $(=3)$. 


\section{Supplementary Information for}

COVID-19 Vaccination Rates Are Lowest among Political Outsiders in the United States

Byungkyu Lee* and James Chu

*Email : $\underline{\text { bl11@indiana.edu }}$

\section{Data Description and Construction}

Data are drawn from the Understanding America Survey (UAS), a probability-based internet panel of American households. Beginning in March 2020, the UAS started a high-frequency longitudinal data collection related to the COVID-19 pandemic, called Understanding Coronavirus in America tracking survey. A subset of the UAS panel was invited to participate in biweekly surveys about the COVID-19 pandemic starting from April 1, 2020. Respondents were assigned to a specific invitation day at random, and invitations were sent out over a 14-day period. This invitation period has been doubled since February 17, 2021, with invitations being sent out over a 28-day period. Each survey wave gave respondents 14 days to respond after they were invited. Incentives were offered to encourage respondents to complete each survey at a rate of 20 USD per 30 minutes of survey time, in accordance with best practices in survey recruitment. Respondents who completed the survey on the day they receive their invitation received a bonus of one dollar. The UAS panel is constantly expanding through new recruitment batches. New members who consented to participate in the COVID tracking survey were added to the sample on a weekly basis $(1,2)$.

To construct our analytic sample, we used the UAS Covid-19 Survey National Sample Longitudinal File, which contains the 29 waves of the UAS Covid long-form data (March 10, 2020 - July 21, 2021), as well as the UAS 350 survey (Wave 30; September 23, 2021 - October 31, 2021). We dropped the first wave of UAS Covid-19 survey data due to the differences in survey recruitment methods, and because most outcome measures were not available. We merged the UAS Covid-19 data with other UAS political surveys to measure respondents' party registration status. We used all publicly available UAS political surveys (UAS 212, UAS 221, UAS318, UAS 331, UAS 335) to measure respondents' party registration status across different periods ((UAS 212: December 13, 2019 to February 04, 2020, UAS 221: January 15, 2020 to January 28, 2020, UAS 318: November 04, 2020 to December 15, 2020, UAS 331: December 16, 2020 to February 07, 2021, UAS 335: February 18, 2021 to April 30, 2021). We assigned the most recent party registration status to each respondent depending on survey interview dates. Among 9,051 respondents, we could identify 7,814 respondents' party registration information.

Our analytic sample includes 7,814 adults who completed at least one wave of the survey and whose party registration status could be identified. They participated in 21.9 waves out of 29 waves, on average, generating 171,187 respondent-wave observations. Across all waves, the overall participation rate was $75 \%$. We use the final survey weight to calculate weighted means of outcomes for each survey date. We account for the unequal number of survey participation by multiplying the wave-specific survey weight by the inverse of the total number of survey waves per participant. The median number of survey participants per day is about 400 . To account for the small sample size that arises because less than 100 respondents participated in the survey from July 8, 2021 to July 19, 2021, and from October 8, 2021 to October 31, 2021, we assign July 9, 2021 and October 8, 2021 to respondents' survey dates when they participate during those two periods respectively. 


\section{Measurement of Political Outsiders}

In this paper, we mainly use respondents' party registration status to measure political outsider status based on the following questions (note that question wordings slightly differ across different surveys). Specifically, we create a categorical indicator for (a) Republican, (b) Democrat, (c) Independent/something else (No political party (independent), No political party (state does not allow registration by party), Libertarian, Green party, Some other party), and (d) political outsiders ("not registered to vote").

[UAS 212] The next set of questions are about the upcoming primaries and caucuses in advance of the 2020 presidential election, and some issues in the news.

Are you:

1 Certain you are registered to vote in the district where you now live

2 Not certain if you are registered to vote in your district

3 Not registered to vote

4 Not sure if registered to vote or not

[UAS 221] This brief survey focuses mainly on issues which are currently in the news, including the 2020 presidential election. Some questions may be familiar. Thank you for answering them again, we want to be sure we have the most up to date information.

Are you:

1 Certain you are registered to vote in the district where you now live

2 Not certain if you are registered to vote in your district

3 Not registered to vote

4 Not sure if registered to vote or not

[UAS 318]: Regardless of whether you voted in the 2020 presidential election, were you registered to vote at your current address in time to cast a ballot in that election if you had chosen to do so?

1 Yes, I was registered to vote in time for the 2020 presidential election

2 I was not registered to vote in time for the 2020 presidential election

3 Not sure

[UAS 331, UAS335]: Regardless of whether you voted in the November 3 election, were you registered to vote at your current address in time to cast a ballot in the November 3 election if you had chosen to do so?

1 Yes, I was registered to vote in time for the election

2 I was not registered to vote in time for the election

3 Not sure

[UAS 212, UAS 221, UAS 318, UAS 331, UAS 335]: Are/were you registered as:

1 Democrat

2 Republican

3 No political party (independent)

4 No political party (state does not allow registration by party)

5 Libertarian 
6 Green party

7 Some other party

Alternatively, we define political outsiders as those who answer "I don't think of myself that way" to the following ideology question, and we still get similar results (available upon request).

[UAS 212, 221]: "Regardless of your political registration or affiliation, where would you place yourself on the political spectrum from extremely liberal to extremely conservative?"

1 Extremely liberal

2 Very liberal

3 Somewhat liberal

4 Liberal side of moderate

5 Completely moderate

6 Conservative side of moderate

7 Somewhat conservative

8 Very conservative

9 Extremely conservative

10 I don't think of myself that way

\section{Measurement of Outcome Variables}

A. COVID-19 vaccine uptake: The UCAS started to ask respondents if they had got their first dose of the COVID-19 vaccine from December 23, 2020, and onward. We created the indicator for COVID-19 vaccination from that question, which equals 1 for respondents who received at least a first dose, and 0 otherwise.

B. COVID-19 vaccine hesitancy: We measure COVID-19 vaccine hesitancy using the following question "How likely are you to get vaccinated for coronavirus once a vaccination is available to the public?" Respondents were classed as hesitant (unsure or somewhat/very unlikely to vaccinate) or willing to vaccinate (somewhat likely/very likely to vaccinate or already vaccinated) following Daly et al (3).

C. COVID positive: We identify whether respondents get COVID-19 if they said, they have been tested positive or they received a diagnosis of having the coronavirus from healthcare professionals based on the following two questions.

Have you been tested for coronavirus? If so, what was the result?

1 I have been tested and I tested positive (I had coronavirus)

2 I have been tested and I tested negative (I did not have coronavirus)

3 I have been tested and I do not know the result

4 I have not been tested

Whether or not you have had a coronavirus test, has a doctor or another healthcare professional diagnosed you as having or probably having the coronavirus?

1 Yes

2 No 
D. COVID-19 risk assessment: We measure how respondents assess COVID-19 risk based on the following question: (On a scale of 0 to 100 percent) "If you do get the coronavirus, what is the percent chance you will be hospitalized (spend at least one night in the hospital) from it? If you're not sure, please give your best guess."

E. Trust in the source of information about Coronavirus: We measure the level of trust across six different sources of information using the question, "how much do you trust the following sources of information about the coronavirus (COVID-19)?” The sources include respondents' physicians, CDC, two representative sources of partisan news outlets (FOX and MSNBC), and two presidents (Trump and Biden). We recode the response option into $3=$ fully trust, $2=$ mostly trust, $1=$ somewhat trust, $0=$ do not trust.

\section{References:}

1. A. Kapteyn, et al., Tracking the Effect of the COVID-19 Pandemic on the Lives of American Households. Survey Research Methods 14, 179-186 (2020).

2. P. G. Szilagyi, et al., Likelihood of COVID-19 vaccination by subgroups across the US: post-election trends and disparities. Human Vaccines \& Immunotherapeutics 17, 3262-3267 (2021).

3. M. Daly, A. Jones, E. Robinson, Public Trust and Willingness to Vaccinate Against COVID-19 in the US From October 14, 2020, to March 29, 2021. JAMA 325, 2397-2399 (2021). 